Nermine EL-SHARKAWY

SELBST(ER)FINDUNG IN DER FREMDE.

TRANSKULTURELLE ASPEKTE IN HUSSAIN AL-MOZANYS ROMAN „MANSUR ODER DER DUFT

DES ABENDLANDES“

Dieser Beitrag geht von der Annahme aus, dass Selbstfindung ein Prozess ist, der transkulturelle Elemente aufweist. Selbstfindung ist der Versuch des Menschen, sich in seinen Eigenheiten und Zielen, v.a. in Abgrenzung von der Gesellschaft und ihren Einflüssen, $\mathrm{zu}$ identifizieren. Diese Art Identitätsfeststellung steht in einem Wechselspiel von Dazugehören und Abgrenzen. Sie stellt das Selbstverständnis des Individuums in Frage. Wechselt man die ursprüngliche Heimat, ob freiwillig oder gezwungen, so spitzt sich die Situation zu, da Fragen nach der nationalen Identität und kulturellen Identität miteinbezogen werden. Begriffe wie Hybridisierung und Transkulturalität spielen hier eine entscheidende Rolle. Inwiefern ist die Selbstfindung bzw. Selbsterfindung in der Fremde transkulturell ist, möchte ich anhand von Hussain Al-Mozanys 2002 erschienenem Roman Mansur oder der Duft des Abendlandes im Folgenden untersuchen. Beginnen werde ich mit dem theoretischen Kontext, in dem mein Beitrag zu situieren ist. Darauffolgend, gehe ich analysierend auf den Inhalt des Romans ein. Die transkulturellen Elemente werden bei jeder Station herausgerabeitet, die die Hauptfigur Mansur mit seiner hybriden Identität durchmacht. Schließlich behandle ich die Problematik der Selbst(er)findung in der Fremde, wie sie im Roman zum Vorschein gekommen ist.

\title{
Theoretischer Kontext
}

Transkulturalität ist der Versuch, im Rahmen gesellschaftlicher, politischer und auch bildungspolitischer Entwicklungen angesichts von Globalisierung und zunehmender Zeit-Raum-Verdichtung kulturelle Selbstverständlichkeiten in 
Frage zu stellen und über traditionelle Vorstellungen von Kultur hinauszuweisen. Bereits 1984 hat Schöfthaler auf die Notwendigkeit einer transkulturellen Erziehung hingewiesen und zur Diskussion über „kulturelle Identität“ und "nationale Identität" beigetragen. ${ }^{1}$ Nationalkulturen als Einheiten von Territorium, Abstammung, Staatlichkeit, Sprache, und gemeinsamer Tradition waren jedoch meist weniger historische Realitäten als vielmehr ideologische Wunschgebilde. Gegenwärtig trifft auf die meisten Staaten zu, dass sie im Prinzip multikulturell und transkulturell bzw. hybrid strukturiert sind. ${ }^{2}$ Lüsebrink zählt die Hybridisierung von Kulturen zu den Charakteristika der aktuellen Phase der Globalisierung. Hiermit ist die zunehmende gegenseitige Beeinflussung und Vermischung der Kulturen des Globus gemeint, die auf Kulturkontakt, Kulturaustausch und Kulturtransfer in ihren vielfältigen Ausprägungen beruht, so dass die vom Nationalismus des 19. Jhd.s geprägten Vorstellungen von Nation und Nationalkultur aufgehoben werden dürfen. ${ }^{3}$ Weiterhin sind Kulturen durch den Prozess der Globalisierung in Folge von Migrationsprozessen, von globalen Verkehrs- und Kommunikationssystemen und ökonomischen Abhängigkeiten auf der Makroebene extern durch weltweite Verflechtungen, Nationalgrenzen überschreitend vernetzt und im Sinne einer interdependenten Globalkultur miteinander verbunden. ${ }^{4}$ Globalisierung geht so einerseits mit Formen der

$1 \quad$ Schöfthaler, Traugott 1984: Multikulturelle und Transkulturelle Erziehung: Zwei Wege zu kosmopolitischen kulturellen Identitäten. In: Flechsig, Karl-Heinz (2002): Kulturelle Identität als Lernproblem. In: Globalisierung als Herausforderung der Erziehung. Theorien, Grundlagen, Fallstudien. Christoph Wulf/ Christine Merkel (Hrsg.), Waxmann Verlag, Münster, S. 67.

Flechsig, Karl-Heinz 2002: Kulturelle Identität als Lernproblem. In: Globalisierung als Herausforderung der Erziehung.Theorien, Grundlagen, Fallstudien. Christoph Wulf/ Christine Merkel (Hrsg.), Waxmann Verlag, Münster, S. 66.

3 Lüsebrink, Hans-Jürgen 2011: Interkulturelle Kommunikation im Kontext der Globalisierung. Herausforderungen, Konzepte, methodische Ansätze. In: Interkulturelle Kommunikation. Perspektiven einer anwendungsorientierten Germanistik. Eckehard Czucka/Eva Neuland (Hrsg.). Iudicium Verlag. München 2011, 34f.

4 Welsch, Wolfgang 1995: Transkulturalität. Zur veränderten Verfasstheit heutiger Kulturen: http://www.forum-interkultur.net/uploads/tx_textdb/28.pdf. Siehe auch Welsch 2010 u. 2011: 
Vereinheitlichung bzw. Homogenisierung individueller Lebensformen und andererseits mit kultureller Diversität einher, so dass sich die Grenzen zwischen Eigenem und Fremdem zunehmend verwischen. Diese Transformation von kulturellen Identitäten ist einer der zentralen Schwerpunkte, mit denen sich die Migrationsliteratur beschäftigt. Schmitz zufolge wird in Deutschland seit 1989 das Leben mit der Fremde und den Fremden als Teil der eigenen Kultur wahrgenommen:

„Es scheint so, als ob die Deutschen erst jetzt, nachdem die 1989 vereinigten beiden Teilstaaten wieder an die nationale Tradition hätten anknüpfen können, begreifen, dass der Staat sich neu erfinden muss. Die alten Konzepte des Nationalen, so heftig sie auch in den 1990er Jahren in der deutschen Öffentlichkeit diskutiert wurden, sind erodiert. Der Staat ist damit freilich nicht überflüssig geworden, aber seine Legitimation, Sinnversprechen und Werte wandeln sich hin zu einer Zukunft, die nicht auf die nationale Tradition allein verpflichtet bleibt. Im Literarischen gar waren die Ansprüche der Naionalliteratur schon längst zweifelhaft und fragwürdig geworden.“5

In einer ihrer Erzählungen spitzt Emine Sevgi Özdamar die Frage nach einer authentischen Identität in der folgenden Äußerung zu: „Ein echter Berliner ist aus Berlin. Ein echter Türke ist aus Kreuzberg. ${ }^{" 6}$ Dieses Beispiel zeigt, wie sich die mit einem Migrationshintergrund Literaturschaffenden auf der Bandbreite zwischen dem Anspruch auf Authentizität und deren Dekonstruktion bzw. Hybridität positionieren. ${ }^{7}$ Eine avancierte Migrationsliteratur versteht, wie Blioumi feststellt, die Migration nicht als Verlust oder Leid, sondern als Stärke, und diese Stärke schöpft aus dem Oszillieren zwischen dem Fremden und dem

\footnotetext{
Was ist eigentlich Transkulturalität? http://www2.unijena.de/welsch/papers/W_Welsch_Was_ist_Transkulturalit\%C3\%A4t.pdf (Stand 12.9.2015).

5 Schmitz, Walter 2011: Demolierter Orientalismus. Über Hussain Al-Mozany, einen deutschsprachigen Exilschriftsteller aus dem Irak. In: Parallelwelten. Thelem. Dresden. S. 179

Özdamar, Emine Sevgi 2010:, Mutter Zunge, Rotbuch, S. 84.

Konuk, Kader 2001: Identitäten im Prozeß. Literatur von Autorinnen aus und in der Türkei in deutscher, englischer und türkischer Sprache. Verlag die blaue Eule, Essen. S. 125.
} 
Eigenen. ${ }^{8}$ In diesem Verständnis drückt das Konzept der Transkulturalität die zunehmende Verflüssigung der binär gedachten Kategorien von kulturell Eigenem und kulturell Fremden, sowie die daraus resultierende Bildung von grenzüberschreitenden Netzwerken aus. Das Konzept der Transkulturalität trägt, nach Welsch, in der theoretischen Perspektive zu einer Auflösung bzw. „Entschärfung“ von kulturellen Differenzen bei, indem diese als temporäre, durchlässige und heterogene Phänomene betrachtet werden. ${ }^{9}$

Hierfür bietet sich als treffendes Beispiel aus der Migrantenliteratur arabischstämmiger Autoren ${ }^{10}$ das Werk von Hussain Al-Mozany. ${ }^{11}$ Im Folgenden

$8 \quad$ Blioumi, Aglaia 2002: Interkulturalität und Literatur. Interkulturelle Elemente in Sten Nadolnys Roman „Selim oder Die Gabe der Rede“. In: Blioumi, Aglaia (Hrsg.): Migration und Interkulturalität in neueren literarischen Texten. Iudicium Verlag. München. S. 28

Welsch 2011: Was ist eigentlich Transkulturalität? http://www2.unijena.de/welsch/papers/W_Welsch_Was_ist_Transkulturalit\%C3\%A4t.pdf . (Stand 12.9.2015).

Nach Arig Saleh, die die Generationen der in Deutschland lebenden, literaturschaffenden arabischen Autoren unterschieden hatte, gehört Al-Mozany der ersten Generation an. Diese erste Generation bezieht sich auf Autoren, die in den 40er und 50er Jahren des 20. Jh.s. geboren wurden. Sie sind überwiegend eine Gruppe aus Exilierten, die auf Grund der politischen Verhältnisse ihre Herkunftsländer verlassen mussten und vielfach in der Bundesrepublik Asyl suchten. Vgl. Saleh, Arig, 2011: Rezeption arabischer Migrationsliteratur in Deutschland. Eine Untersuchung am Beispiel der in Deutschland lebenden syrischen Autoren. Inauguraldissertation zur Erlangung des Grades eines Doktors der Arabistik am Fachbereich Geschichts- und Kulturwissenschaften der Freien Universität Berlin. S. 17, 20, 49 u. 93.

Al-Mozany wurde 1954 in Amarah geboren und wuchs in Bagdad auf. Nach journalistischen Tätigkeiten im Libanon wechselte er 1980 ins bundesdeutsche Exil. Er studierte Arabistik, Islamwissenschaft, Germanistik und Publizistik an der Westfälischen Wilhelms-Universität und schloss sein Studium mit einer Arbeit über den ägyptischen Autor Nagib Mahfuz ab. Er lebt heute als freiberuflicher Schriftsteller und Übersetzer in Berlin. Al-Mozanys Übersetzungsleistung ist beeindruckend. Er übertrug Werke u.a. von Nicolas Born, Günter Grass, Robert Musil, Rainer Maria Rilke, Walter Benjamin, Elias Canetti, Hans Georg Gadamer und Jürgen Habermas ins Arabische. 1999 veröffentlichte er seinen ersten auf Deutsch verfassten Roman Der Marschländer. Sein zweiter erschien 2002 unter dem Titel Mansur oder der Duft des Abendlandes und sein dritter Roman Das Geständnis des Fleischhauers erschien 2007. Erwähnenswert ist, dass Al-Mozany 2005 das Jugendstück Klara und Abbas veröffentlicht hatte, das bis heute auf verschiedenen Bühnen aufgeführt wird. Hussain Al-Mozany erhielt 2003 den Förderpreis zum Adelbert-von-Chamisso-Preis. 2009 übernahm er die Chamisso-Poetikdozentur an der TU Dresden. 
gehe ich analysierend auf das Werk ein, wobei ich die transkulturellen Aspekte des Romans hervorheben werde, um schließlich die Schnittstelle zwischen Selbst(er)findung und Transkulturalität zu beleuchten.

\section{Mansur oder Der Duft des Abendlandes}

Auf eine Frage der Berliner Tageszeitung, ob er sich in Deutschland so richtig zu Hause fühle, antwortete Hussain Al-Mozany mit nein. In seinem „Heimat aus Wörtern“ betitelten Artikel diskutierte er diesen Gedanken weiter und verband ihn mit der Frage nach der Identität bzw. der Identitätssuche:

„Bin ich in Deutschland zufrieden? Nein. War ich, bevor ich nach Deutschland gekommen bin, zufrieden? Ebenfalls Nein. Also liegt diese Unzufriedenheit in mir selbst begründet und nicht bei den Deutschen, Arabern oder Muslimen. [...] Wissen Sie ich strebe nicht nach einer Identität.Ich suche auch keine Identität, und wenn ich auch überhaupt eine Identität suche, dann suche ich die nicht bei einer Nation - auch nicht bei den Irakern. ${ }^{\text {"12 }}$

Al-Mozany besteht darauf, für sich eine freiwillige Identität zu wählen:

„Dass ich als Iraker, Araber, Muslim, Schiit geboren bin, habe ich nicht bestimmt, aber ein deutscher Schriftsteller zu sein, ohne den deutschen Nationalstolz zu hegen, das wollte ich allemal. Denn die deutsche Sprache gehört weder Deutschland noch sonst einem anderen Land, sondern steht allen Menschen überall frei zur Verfügung. “13

Diese freiwillig gewählte Identität konstruiert Al-Mozany aus der Literatur. Seine Einstellung korrespondiert mit den Ausführungen Stuart Halls über kulturelle Identitäten, die er für „die instabilen Identifikationspunkte oder Nahtstellen, die innerhalb der Diskurse über Geschichte und Kultur gebildet werden. Kein Wesen, sondern eine Positionierung." "14 Al-Mozanys Werk wird durch das Leben zwischen zwei Kulturen, die Erfahrung von Krieg, Terror und

12 Al-Mozany, Hussain 2011: Parallelwelten.Thelem. Dresden. S. 85f.

13 Ebd. S. 88.

14 Hall, Stuart 1994: Rassismus und kulturelle Identität. In: Konuk, Kader: Identitäten im Prozeß. Literatur von Autorinnen aus und in der Türkei in deutscher, englischer und türkischer Sprache. Literaturwissenschaft in der Blauen Eule. Band 28. Essen. S. 131. 
Flucht geprägt. Wo ist eigentlich die Heimat? Die alten Kategorisierungen von „Heimat" und „Fremde“, „Eigenem“ und „Anderem“ verlieren an Trennschärfe. Hierin kommt eine Darlegung von Wolfgang Welsch hervor: „Kulturen sind intern durch eine Pluralisierung möglicher Identitäten gekennzeichnet und weisen extern grenzüberschreitende Konturen auf. Sie haben eine neuartige Form angenommen, die durch die klassischen Kulturgrenzen wie selbstverständlich hindurchgeht. ${ }^{\prime 15}$ Gerade hierin liegt das Konzept der Transkulturalität. Gerade die veränderte Auffassung der Kulturen versucht Al-Mozany in seinem Euvre literarisch zu verarbeiten und zu fragen, wie unser Verhältnis zur Fremde in der Welt aussehen könnte. Al-Mozanys Romandebüt Der Marschländer (1999) thematisiert den Transit und die Flucht aus der irakischen Diktatur. Die Folgeromane Mansur oder der Duft des Abendlandes (2002) und Das Geständnis des Fleischhauers (2007) behandeln die aktuellen Konflikte im Irak und das deutsch-arabische Verhältnis. Bemerkenswert ist, dass jeder der drei Romane eine Facette des Erfahrungsspektrums von Exil und Fremde ausleuchtet. Mit Witz und Ironie ${ }^{16}$ erzählt Al-Mozany die Geschichte des jungen, um Asyl bittenden Mansur, der behauptet, er stamme von einem deutschen Kreuzritter ab. Mansur desertiert

Welsch, Wolfgang 1995: Transkulturalität. Zur veränderten Verfasstheit heutiger Kulturen. In: Lüsebrink, Hans-Jürgen 2008: Interkulturelle Kommunikation. Interaktion, Fremdwahrnehmung, Kulturtransfer. 2., aktualisierte und erweiterte Auflage. Verlag J. B. Metzler. Stuttgart/ Weimar, S. 18.

Halter nennt das Werk einen Schelmenroman. Vgl. Halter, Martin 2002: Spätaussiedler aus Bagdad. Abendland gesucht: Ein Schelmenroman von Hussain Al-Mozany. In: Frankfurter Allgemeine Zeitung, 12.9.2002. Fridolin kategorisiert es als eine Satire. Vgl. Furger, Fridolin 2002: Flucht aus Krieg und Diktatur. Hussain Al-Mozany schreibt mit „Mansur oder der Duft des Abendlandes" eine wunderbare Satire auf die deutsche Asyl- und Einbürgerungspraxis. In: Der Bund, 25.5.2002. Stefan Weidner sieht den Humor als die größte Leistung von Al-Mozanys Roman: „Er umfasst alle Spielarten, die Parodie, die Groteske, die Satire, die Anekdote ebenso wie Sprachwitz und Ironie.“ Weidner, Stefan 2002: Zwischen Festmahl und Furz. Hussain AlMozany sucht den Deutschen im Araber. In: Die Zeit, 23.5.2002. Diese Gattungen wurden von Hofmann unter den literarischen Gestaltungsmitteln gezählt, die die Infragestellung starrer Normsysteme mit den Mitteln der Sprache und der Fiktion ausdrücken, welche die Erfahrungen von poetischer Alterität bewusst machen. „Die Groteske zeigt die übertriebene Darstellung einer Person oder einer Sache, wobei deutlich wird, dass gegebene Konventionen verletzt werden; die Verletzung dieser Konventionen kann durch den fremden Blick motiviert sein.“ Hofmann, S. 59. 
in seiner Heimat Irak aus der Armee und flüchtet vor Verfolgung und Krieg nach Deutschland. Hier will er mit Hilfe einer alten Urkunde seinem Anspruch auf die deutsche Staatsbürgerschaft Geltung verschaffen: seine muslimische Urahnin Aischa, habe vor gut tausend Jahren den fränkischen Kreuzritter und Wanderprediger Peter der Einsiedler geheiratet. Was als kleiner Fall eines idealistischen Anwalts in Köln beginnt, entwickelt sich im Verlauf der Geschichte zum medienträchtigen Skandal. Im Schlagabtausch eines skeptischen, doch wohlwollenden Richters mit dem Protagonisten wird die deutsche Realität aus ungewöhnlicher Perspektive geschildert. Mansur muss sich seltsamen Untersuchungen an Leib und Seele unterziehen, um seine Behauptung zu beweisen. Unterdessen lernt der junge Mann im Asylantenheim Menschen aus verschiedenen Ländern kennen, die ihn mit dem Leben in der Fremde und der käuflichen Liebe vertraut machen. Wie sich aus der Fabel des Romans hervorhebt, verläuft die Handlung hier auf drei Schienen:

- Die Beschreibung der Flucht Mansurs aus dem Irak über Syrien, bis er in Deutschland ankommt;

- Die Beschreibung seines Lebens als Einwanderer in Deutschland sowie seine Klage vor Gericht;

- Die Geschichte von Aischa und Peter dem Einsiedler.

Jede der erzählten Ebenen unterhält ihr eigenes Figurenensemble, wobei immer wieder Al-Mozanys Versuch klar wird, Bezugs- bzw. Anschlusspunkte zwischen Deutschland und dem Irak zu konstatieren, um ans Ziel zu gelangen, „den Deutschen im Araber" ${ }^{\text {"17 }}$ zu entdecken. Die Erzählweise ist nicht linear. Die Mosaiksteine jeder Ebene lassen sich durch Rückblende und Vorausblick zu einem gemeinsamen Bild zusammenfügen. Bevor ich im folgenden jede dieser Ebenen behandle, wobei die transkulturellen Aspekte der jeweiligen Ebene unterstrichen werden, scheint es mir wichtig, dem hier angewandten Erzählmodell einige Worte zu widmen. Genette, Martinez und Scheffel folgend will ich das Problem der Perspektivierung des Erzählten unter dem Begriff der Fokalisierung fassen. Dies konzentriert sich auf die Frage, aus welcher Sicht das

$17 \quad$ Weidner, Stefan: a.a. O. 
in einer Erzählung Erzählte vermittelt wird. Hierin unterscheidet man drei Typen: Nullfokalisierung, interne Fokalisierung und externe Fokalisierung. ${ }^{18}$ In AlMozanys Roman ist die Darstellung des Geschehns so eindeutig auf die Perspektive der wahrnehmenden Figur Mansur konzentriert, dass wir von einem typischen Fall von interner Fokalisierung sprechen können. ${ }^{19}$ Nach Genette werden die Begriffe interne Fokalisierung, aktorial und Mitsicht mit gleicher Bedeutung verwendet. ${ }^{20}$ Sie bezeichnen, dass das Erzählte eng an die Wahrnehmung der Figur eng gekoppelt wird. Der Erzähler sagt nicht mehr, als die Figur weiß. Schon am Anfang des Romans lässt das im Text Geschilderte Rückschlüsse auf denjenigen zu, der es wahrnimmt, nämlich die Hauptfigur Mansur:

„In einer der weißen Stunden der Abdendämmerung machte sich der Fremde Mansur auf den Weg. Das Licht des Mondes überflutete alles Irdische und so war es leicht, Magnolienblüten, Teerosen und Orchiden abzupflücken, glühende Lichtperlen, die aus wundersamen Träumen zu stammen schienen. Man konnte die weißfedrigen Lärchen sehen, die Türme einer Kirche aus rotem Ziegelstein und dort, unweit jener Kirche am linken Ufer des grauen Flusses, lag hinter den Reihen junger Birken- und Tannenbäume der alte Friedhof, auf dem die Gebeine des heiligen Kriegers angeblich ruhten. Hier nahm die Geschichte des Einsiedlers ihren Lauf. “21

Insbesondere gilt dieses Erzählmodell, wenn weit über die optische Perspektivität hinaus auf subjektive Faktoren wie Informationsstand und kognitive, ideologische oder psychologische Wahrnehmungs- und Bewertungsmuster erzählt werden.$^{22}$ Der Roman bietet viele Beispiele dafür an,

18

Martinez, Matias/ Scheffel, Michael 2009: Einführung in die Erzähltheorie. 8. Aufl. C.H.Beck, S. $64 \mathrm{f}$.

Präziser können wir auch von fixierter interner Fokalisierung sprechen, da die Wahrnehmung während des ganzen Textes an die Figur Mansurs gebunden bleibt. Vgl. Mair, Meinhard 2015: Erzähltextanalyse. Modelle, Kategorien, Parameter. Ibidem Verlag, Stuttgart, S. 219.

Martinez/Scheffel, a. a. O.

Al-Mozany, Hussain 2002: Mansur oder der Duft des Abendlandes. Reclam, Leipzig, S. 6. Darauf verwiesen mit der Sigle M.

Vgl. Nünning, Ansgar 2001: Metzler Lexikon. Literatur- und Kulturtheorie:Ansätze-PersonenGrundbegriffe. 2.überarb. und erw. Aufl., Stuttgart,Weimar, S. 186f. 
wie das folgende Beispiel, das die problematische Beziehung Mansurs zu seiner ursprünglichen Heimat zeigt, die er nun als „fernes Land“ bezeichnet, nachdem ihm gerichtlich die deutsche Staatsangehörigkeit gewährt wurde:

„Vergangen diese Heimat, vorbei die Schandtaten in fremden Gebieten, die ewigen Kränkungen - jenes ferne Land versank samt seinen Kriegen, seiner despotisch angelegten Hinhaltetaktik und seiner Aushungerungspolitik, samt den Massengräbern, den gefeierten Mördern und Massenmördern immer tiefer in Erinnerung. [...] Mansur sah sich arabischsprachige Bücher zu einem Häufchen schichten, das er noch vor der sich anbahnenden Hochzeit als Buße auf einem kirchlichen Altar zu entzünden hatte.“ (M 253)

Nun möchten wir Mansur auf seiner Suche nach sich selbst begleiten, um zu prüfen, ob ihm tatsächlich gelungen war, für sich eine neue Heimat und eine wahre Identität zu finden bzw. zu erfinden.

\section{Die Geschichte von Aischa und Peter dem Einsiedler}

Versuchen wir die Geschichte chronologisch zu rekonstruieren, so finden wir heraus, dass die Erzählung von Peter dem Einsiedler bzw. Petrus von Amiens (Petrus der Eremit; †8.7.115), die ihren Beginn im mittelalterlichen Frankreich nimmt und sich über Deutschland bis Mesopotamien erstreckt, als Vorgeschichte der Geschichte Mansurs fungiert. Wie Tausende anderer Europäer zur Kreuzfahrerzeit zog Peter nach Palästina, um den Muslimen das Grab Christi zu entreissen. Die Quellen erwähnen, dass Peter nach dem Aufruf des Papstes zum Initiator und Anführer des sogenannten Volkskreuzzugs wurde, an dem sich Tausende begeisterter, jedoch militärisch unerfahrener Abenteurer beteiligten. Er zog dann über Aachen nach Köln, wo sich Tausende Anhänger in seinem Gefolge befanden. Mit diesen erreichte er auch Jerusalem 1099, spielte jedoch keine Führungsrolle mehr. Nach Beendigung des Kreuzzugs kehrte er nach Frankreich zurück und wurde Mitbegründer und Prior des Augustinerklosters Neufmoutier. „In der Legende wurde Peter zum eigentlichen Auslöser des ersten Kreuzzuges, da er ein Hilfsgesuch des Patriarchen von Jerusalem nach Rom gebracht habe. ${ }^{\text {“23 }}$

23 Heymer, Björn 1994: Biographisch-bibliographisches Kirchenlexikon. Band VII. Bautz Verlag, Nordhausen. Spalten 330-331. S. a.: https://de.wikipedia.org/wiki/Peter_der_Einsiedler. (Stand 12.9.2015) 
In Al-Mozanys Version allerdings sollte sich Peter in Aischa verlieben und sie heiraten. Diese Geschichte setzt nicht nur einen Dialog über Orient und Okzident $^{24}$ in Gang, sondern weist auch auf den literarischen und historischen Wert solcher interkulturellen Begegungen hin, die vor einem kriegerischem Hintergrund stattgefunden hatten. ${ }^{25}$ Die Unterstützung Karls des Großen in seinem Krieg gegen die heidnischen Völker durch Harun al-Raschid wird als erstes Begegnungsmoment zwischen Orient und Okzident rekapituliert. (M 62) Auf dieses folgen andere Geschichten, die den kulturellen, wissenschaftlichen, medizinischen und militärischen Vorzug der damaligen Araber den Franken gegenüber zeigen (M 63). Weitere (kriegerische) Begegnungen zur Zeit von Friedrich Barbarossa werden ebenfalls erwähnt (M 179). Welsch verwendet den Begriff der Transkulturalität, um die aktuelle hybride Verfasstheit von Kulturen zu beschreiben. Es liegt m. E. aber auf der Hand, dass Transkulturalät auch historisch verfolgt werden konnte. Hierfür stellen sich Mythen, Religionen, Volkserzählungen, -lieder u.ä. als übergeordnete Kategorien dar, in denen sich Elemente aus verschienden Kulturkreisen miteinander vermischen und

Andreas Pflitsch zufolge bezeichnet der Begriff „Orient“ die Gesamtheit der Ideen, die sich der Westen vom Osten gemacht hat. „Es handelt sich weniger um geographische Grenzen oder die Grenzen zwischen real existierenden kulturellen Unterschieden, als vielmehr eine willkürlich gezogene Grenzlinie zwischen dem Vertrauten und dem Fremden, zwischen „uns“ und „den Anderen". Dementsprechend sollte man den Orient nicht auf der Landkarte, sondern in den Köpfen suchen. Er kommt zum Ergebnis, dass der Orient ein abendlänischer Mythos ist. Ebenfalls bezeichnet m. E. das von Al-Mozany im Romantitel benutzte Schlagwort „Abendland“ weniger einen geographischen Begriff, als eine geschichtspolitische und kulturphilosophische Chiffre. Hierin folgt er einer romantischen Tradition. Das Wort entstand 1529 in Anlehnung an das vom Martin Luther eingeführtes Morgenland. Der vorliegende Roman impliziet, dass sich der Begriff Abendland als fiktiver Konstrukt herausstellen lässt. Vgl. Pflitsch, Andreas: Kreidestriche um die Fruchtsamkeit zu narren? Orientierung über den abendländischen Mythos vom Orient. S. 8 und Repgen, Konrad: Was war das Abendland - Eine Begriffsgeschichte, S. 14. In: Haridi, Alexander (Hrsg.): West-östlicher Seiltanz. Deutsch-arabischer Kulturaustausch im Schnittpunkt Kairo. DAAD. Trio. Bonn. 2005.

Lüsebrink hat auf die „konfliktuelle Dimension“ als ein Charaktersitikum aller Globalisierungsphasen hingewiesen, bevor er die aktuelle Phase als diejenige ausgezeichnet, die durch konfliktuelle Bedeutung kultureller Phänomene am stärksten betroffen untersteicht. Lüsebrink 2011, a. a. O. S. 35. 
wechselseitig beeinflüssen. Die Thematisierung des Fremden in den eigenen Texten und vice versa stellt heraus, wie das Eigene nur im Bezug auf das Andere und das Fremde bestimmt werden konnte. ${ }^{26}$ Dieses Element greift Al-Mozany im ganzen Roman auf, v.a. aber in den historischen bzw. historisierenden Stellen, um die Bausteine einer nationalen Identität zu dekonstruieren und hervorzuheben, dass die sogenannte nationale Identität bereits immer mit fremden Elementen verflochten gewesen war, wie das folgende Beispiel zeigt:

„Ja, Karl hat zu dieser Zeit mithilfe seines Duzfreundes die Sachsen und das später ausgerottete
Volk der Awaren unterworfen. Und selbstverständlich hat er sich dabei von Anfang an der
Kriegskunst seiner arabischen Verbündeten bedient. Im Namen Gottes hat er das Schwert
erhoben und viele heidnische Völker bekehrt. Aus Dankbarkeit bekam Harun das so genannte
Friesische Manteltuch geschenkt:
Kleide schenk ich den Meinen,
gefärbt mit friesischen Farben,
welche du, Harun, wohl nie
hattest mit Augen gesehen.“ (M 63)

\section{Mansur auf der Flucht}

Der Roman Al-Mozanys beginnt, als der Titelheld bereits aus der irakischen Armee desertiert war. Auf der Flucht begegnete ihm eine verwitwete junge Frau. Diese brachte ihn auf die Idee, sich auf die Reise nach Deutschland zu begeben, denn sein Gesicht ähnelte den Gesichtern der Deutschen. In seinem Gedächtnis versuchte er nun Erinnerungsmomente an die deutsche Kultur zu sammeln und entdeckte, dass eine allererste Begegnung mit dieser durch die Lektüre einer arabischen Fassung von Goethes Leiden des jungen Werther gelungen war (M 10). Er merkte, dass die Bierproduktion in Bagdad vollständig in deutscher Hand lag. Sogar die berühmte Bagdadbahn wurde durch Kaiser Wilhelm eröffnet, der mit seinem langen, an beiden Seiten hochgezwirbelten Schnurrbart zum Verwechseln einem Beduinenscheich ähnelte. Deutschland erschien Mansur wie ein verlorenes Paradies zu sein, das sich aus verschiedenen Gründen tief in seinem Gedächtnis eingraben konnte.

$26 \quad$ Vgl. Hoffman, Michael 2006: a.a.O., S. 69. 
„Es war im Grunde Frontgeplauder, die letzten Worte eines sterbenden Soldaten, die das Deutschland Peters betrafen, wo alles so schön ordentlich, sauber, grün und satt aussehen sollte. Demnach war jenes Land ein gepflegter freier Staat, dessen Menschen im Glück schwammen, dessen Frauen von außergewöhnlicher Schönheit, Grazie und Attraktivität waren und vor allem ansprechbar. Während der Besatzungszeit, so wusste der Kamerad zu berichten, seien sie manchmal für eine Schachtel Zigaretten zu haben gewesen. Deutschland insgesamt sei ein riesiger Eintopf, in dem alles, wirklich alles stecke und sich vermische und zwar zur rechten Zeit gar genossen werden müsse. Es gebe Gemüse und viel Fleisch darin und jeder dürfte davon schlürfen, so viel es ihm gefalle und sei es bis zur Übersättigung; niemand könne dort Hungers sterben. Aber wehe dem, der zu spät komme, er werde ein Opfer seiner Trägheit und Unentschlossenheit, denn der Eintopf sei dann zur Neige gegangen.“ (M 22)

Um Anteil an diesem internationalen Eintopf zu bekommen, begann Mansur, nach anfänglichem Zögern, seinen Fluchtplan nach Deutschland auszuarbeiten. Um sich auf das Vorhaben vorzubereiten, kaufte er Bücher über Deutschland und die deutsche Sprache vom Suq as-Saray, wo sich die antiquarischen Buchläden befanden, besorgte sich einen falschen Pass mit falschem Visum und überquerte die Grenze zwischen dem Irak und Syrien. Von dort nahm er die Maschine, flog nach Deutschland und landete im Kölner Flughafen. Bereits am Bord des Flugzeugs, begann die desertierte Heimat, sich zu verflüchtigen, sodass ihre Schwere Mansurs Gemüt nicht mehr bedrückte:

„Der arabische Halbmond war in die Ferne gerückt, der fliegende Teppisch ausgeklopft (...). Die syrische Lufthoheit war längst überwunden und unter ihnen lagen, wie die kartographische Aufschlüsselung auf dem Monitor zeigte, die letzten Bergzüge des Antilibanon, wo einst die furchtbaren Schlachten zwischen Kreuzrittern und islamischen Kriegern stattgefunden hatten und sich auch jüngst die Ritter der Klagemauer mit den Kämpfern der Partei Gottes immer wieder Scharmützel lieferten. All das zog von hier aus schnell vorüber, wurde zur Geschichte getan und löste sich in der Erinnerung in einzelne Farbstreifen auf. Wie schön wäre es, wenn man immer von so weit oben blicken könnte. Alles wirkte klein und kleiner, bis das Meer es verschluckte.“ (M 45)

Mit bescheidenen Englischsprachkenntnissen begann er nun, sich eine Existenz in der selbstgewählten Heimat zu verschaffen. 
Selbst(er)findung in der Fremde

\section{Mansur in Deutschland}

Mansurs Landung im Kölner Flughafen nimmt er als Heimkehr wahr: „Das war Deutschland, die alte Heimat der lang verkannten Vorfahren“ (M 50) und weiter noch: „Mit den Zähnen sollte er sich in deutschem Boden festbeißen. Niemand konnte ihn wieder aus dem Land der christlich-kriegerischen Franken vertreiben!“(M 52). In Deutschland half ihm sein Landsman Said, der als Übersetzer und Dolmetscher arbeitete, einen Platz in einem Asylantenheim in Köln zu finden. Auch war Said derjenige, der Mansur mit dem Rechtsanwalt Knosper bekannt machte. Nach Plädoyers, Auseinandersetzungen, Gentests und ausgedehnten Recherchen gelang es Knosper, Mansur als Deutschen zu rehabilitieren. Folglich wollte er ihn mit der deutschen Lisa verheiraten.

Aber viel früher traf Mansur in einem sogenannten „Hauptquartier der Verstoßenen“ verschiedene Arabischstämmige: Libanesen, Palästinenser und Iraker. Sie trafen sich in einem Tanzlokal, das von einer sogenannten Mutter der Araber, einer deutschen Frau, geführt wurde, um zu saufen, tanzen und arabische Musik zu hören. Immer wieder diskutierten sie über die Zustände in der arabischen Welt und stritten sich dabei mit scharfer Zunge. Allerdings verstanden sie es, Eskalationen durch Witz und Akzeptanz zu meiden, bevor es zu spät wurde. Erst in der fernen Fremde Deutschlands fühlte sich Mansur als Mitglied einer großen arabischen Familie, die es früher nur in seiner Fantasie oder der Nationalhymne seiner Heimat gegeben hatte (M 78). Ebenfalls lernte er in der Enge des Asylantenheims andere Menschen aus verschiedenen Kulturkreisen kennen, darunter Perser und Inder, die alle zusammenleben mussten. Hier bediente er sich ebenfalls des Englischen als Verständigungsmittel. Recht interessant ist ihre Einstellung der jeweiligen Staatsmännern gegenüber.

„»Khomeni is good?«

$»$ No, Khomeni is not good.«

»Saddam is good? «

»No, Saddam is not good.«

Da Mansur den Staats- oder Bundespräsidenten von Indien nicht namentlich kannte, formulierte er seine Frage allgemein:

$»$ India is good? «

»Yes, oh, very good, but government is not good.« 
$\gg$ And Libanon is good?«

»Yes, but the people are not good!«" (M 83)

Es zeigt sich hier die Unzufriedenheit der Völker aus der Dritten Welt mit ihren herrschenden Regierungen und die universelle Bereitschaft des einfachen Volkes, mit den anderen Frieden zu halten. Trotz der gemeinsamen blutigen jungen Vergangenheit während des achtjährigen Irak-Iran Krieges, haben sich Mansur und die Perser nicht als Erzfeinde behandelt und konnten sogar zusammen koexistieren. An andere Greultaten wurde erinnert, wie die Verfolgung der Juden durch Hitler und den Libanesischen Bürgerkrieg, um die Sinnlosigkeit des Kriegs schlechthin bloß zu stellen und zu verurteilen (M 129).

Der Aufenthalt Mansurs im Asylantenheim endete, als dort ein Brand ausbrach. Der Unfall zeigte das Mißtrauen der meisten Asylanten ihren deutschen Gastgebern gegenüber, was Mansur heftig kritisierte. Dieser Vorfall brachte ihn den Deutschen näher. Mansur ist der Meinung, die Fremden hätten kein Recht darauf, den Deutschen kritisch zu hinterfragen.

„Mit welchem Recht kam ein Fremdling ungefragt und mit dem festen Vorsatz, unter allen Umständen zu bleiben, in dieses Land, um dann einen humanitären Fragenkatalog an die Adresse der nachdenklichen und ständig besorgten Gastgeber zu richten? War das die Gegenleistung für jahrelange kostenlose Verpflegung und Unterbringung? Was könnten die deutschen Menschen dafür, dass Gott sie schön, stark, und reich geschaffen hatte? Ja, hätten sie lieber baren Fußes und in ausgefransten Kleidern wandern sollen, um das Mitleid ihrer Neider zu erregen? Was für eine niederträchtige, barbarische Philosophie!“” (M 204)

Mansur möchte nicht in die Haut eines Opfers schlüpfen und ständig die Anderen für das eigene Leid anklagen. So entschied er sich dafür, sich vom Lokal der Araber zu verabschieden, weil er der größeren Menschheit angehören möchte:

„Was verband ihn überhaupt mit diesen Leuten? Die Sprache, wo doch jeder hier seine eigene Zunge schärfte? Oder seine Haut? Hatte er jemals eine getragen? War er nicht jeglichen Schutzes bloß? Und was war mit seiner Seele? War sie nicht zerbrochen in Stellungsgräben und Grüften? Oder zerquälten ihn letzendlich die fortwährenden Niederlagen, die jeder Araber egal an welchem Ort erfuhr und mit sich schleppte wie Kadaver? Vielleicht stand er unter starkem westlich-jüdischen Einfluss, ohne es zu wissen? Diese verdammte, verklärte, dämonische Rhythmik der heimgesuchten Seele, die sich von der Geschichte, den omayyadischen Mördern, den Osmanen, den weißhäutigen Okzidentalen und wer weiß wem stets misshandelt, zerstoßen 
Selbst(er)findung in der Fremde

und gefährdet fühlte! Aber wo sollten die Araber diese ihre gejagte, misstrauische Seele bergen? Warum konnten er und seinesgleichen nicht einfach den lernfähigen, bodenständigen, den jeglicher ethnischer Fesseln entledigten Geistern, den siegreichen, optimistischen, lebenszugewandten, pragmatischen Menschen angehören?“ (M 222)

Mansur wurde tatsächlich eingebürgert. ${ }^{27}$ Beim Kölner Karneval tanzte und schunkelte er, obwohl ihm die Polonaisen der Jecken so fremd waren wie Kamelkarawanen und das „Alaaf“ seinem feinen $\mathrm{Ohr}$ wie „getrocknetes Viehfutter" klang (M 236). Herr Knosper, sein Rechtsanwalt, ermutigte ihn dazu, in der neuen Heimat Wurzeln zu schlagen und die hübsche Lisa zu heiraten. Diese Mansur-,,Bewunderin“ habe sich bereit erklärt, mit ihm Glück und Unglück zu teilen. Um sich näher kennen zu lernen, traf sich das Brautpaar zum Essen. Die Braut erzählte Mansur, das, was sie nun veranstalte, bilde die Krönung eines bizzaren Witzes: „Allein die Vorstellung, »den alten orientalischen Bock« zu nehmen, sei eine reizvolle Herausforderung. Er, Mansur, könne sicher sein, dass niemand sie dafür habe bestechen müssen.“ (M 260). In einem weiteren Abschnitt berührte Mansur zögernd Lisas Körper, der vollkommen nackt neben ihm lag. Er fühlte sich, am Ende seiner Kräfte angelangt, ausgebrannt und ohne Begehren (M 266). So stieß er mit beiden Füßen heftig die Decke weg und stand auf, legte seine Kleider wieder an und ging leise hinaus (M 270).

Verfolgen wir die Handlung von Mansurs unglaublichem mittelalterlichem Anfang bis zur gegenwärtigen Abenteuern in der deutschen gewählten Heimat, so entpuppt sich allmählich die Frage der Identität als zentrales Thema. Dieses wird in den folgenden Ausführungen behandelt.

27 Vgl. das Göttinger Bericht an das Sonderverwaltungsgericht (Einsiedler-Ausschuss). Fragen danach, was für die abendländische Kultur typisch ist und welchen Einfluss die semetischen Merkmale auf Mansur hatten, werden gestellt, jedoch ohne eine klärende Antwort anbieten zu können. Demzufolge empfiehlt der Bericht Kopromissbereitschaft zu zeigen und Mansur für unbestimmte Zeit auf dem Gebiet des heutigen Deutschlands zu dulden, obwohl sein Fall weitere Untersuchungen notwendig macht. M 239-244. 
Selbst(er)findung in der Fremde

Der Roman wimmelt von einer Fülle von Elementen, die sich aus verschiedenen Kulturkreisen zusammensetzen und sich gegenseitig beeinflussen. Neben den oben erwähnten, manifestieren sie sich beispielsweise in folgenden Formen:

- Propheten und Religionen wie die drei abrahimitischen Reliogionen und ihre Querverbindungen und Wechselbeziehungen;

- Mythen, wie sie im Gilgameschepos vorkamen;

- Sprachen wie Englisch, Arabisch, Deutsch, Russisch, Persisch und Türkisch sowie die Beziehung von Mansur zu diesen Sprachen;

- Politische Führer, Denker, Schriftsteller und Sänger sowohl aus der Vergangenheit, als auch aus der Gegenwart.

All diese Elemente zeugen davon, dass Austauschprozesse und Überlagerungen die Person Mansurs geformt haben. Die binären Kategorien von kulturell Eigenem und kulturell Fremdem verflüssigen sich zunehmend. Kulturelle Differenzen werden aufgelöst bzw. entschärft. Mansur als Individuum ist eigentlich transkulturell. Seine Erfahrung mit den deutschen, aber auch mit den anderen fremden Elementen in sich ermöglichte das Erkennen von Ähnlichkeiten mit äußeren Fremdheiten.

Bereits auf der ersten Seite des Romans wurde der Titelheld als der „Fremde Mansur“ vorgestellt, ein Tatbestand, der seine Entfremdung in der Ursprungsheimat unterstreicht. Erwähnenswert ist hier, dass diese Beschreibung mit den Aussagen Al-Mozanys über seine Identität korrrespondiert:

„Ich bin weder Araber noch Iraker noch Muslim, sondern einfach ein Fremder, und wenn schon ein Deutscher, dann nur im Sinne der Literatur und Philosophie, nicht aber im Sinne des deutschen Grundgesetzes und nicht einmal im Sinne der deutschen Sprache. “28

Eine gescheiterte Liebesbeziehung, ein gespanntes Verhältnis zum eigenen Vater und ein unendlich scheinender Krieg, dessen Ziele er nicht mehr begreifen konnte, machten ihn anfällig für jede sich bietende Chance zur Flucht. Diese

28 Al-Mozany, Hussain 2011: a.a. O. S. $86 f$. 
manifestierte sich zuerst in der äußerlichen Ähnlichkeit Mansurs mit den deutschen Gesichtern (M 8). In einem antiquarischen Buch über Deutschland fand er das Bild eines fünfzigjährigen Deutschen, der verblüffende Ähnlichkeit mit seinem Vater hatte (M 19). Allmählich distanzierte er sich von dem Eigenem und öffnete sich dem Fremden. Darauf wurde die bis dahin anscheinend homogene Identität gebrochen. Eine Art Aushandeln einer neuen, flüssigen Form von Identität begann, die ein „Patchwork“ aufgenommener und bearbeiteter kultureller Perspektiven darstellt. ${ }^{29}$ „Das Subjekt ist Knoten- und Kreuzungspunkt der Sprachen, Ordnungen, Diskurse, Systeme wie auch der Wahrnehmungen, Begehren, Emotionen, Bewusstseinsprozesse, die es durchziehen. ${ }^{“{ }^{30}}$ Kader Konuk weist darauf hin ,dass der innere Kern des Subjekts nicht autonom ist, sondern im Verhältnis zu anderen und der Außenwelt geformt wird.“" ${ }^{\text {31 }}$ Es öffnet sich hier das breite Feld der Aufnahme des Fremden, der Überführung von Fremdem in Vertrautes und auch der Bereicherung des Eigenen durch das Fremde. Hofmann weist darauf hin, dass die Verbindung der Bereitschaft, das Fremde aufzunehmen mit dem Prozess der Selbstfindung schon lange ein Thema der westlichen Literatur gewesen war:

„Das Fremde als das noch Unbekannte bezieht sich auf Nicht-Gewusstes, aber auch auf die Möglichkeit des Wissens und Kennenlernens. Bei dieser Inszenierung von Eigenem und Fremden werden Struktur und Topos der Reise für Ästhetisierung der Selbst- und Fremdbegegnung maßgeblich. Die prototypischen Figuren sind Abenteurer, Entdecker, Forscher, Eroberer, Siedler und Kolonisatoren, die sich aus der Heimat aufmachen, um das Fremde zu suchen. Dass der Mann in die Fremde muss, um die Mission seiner Selbstfindung zu erfüllen, ist als wirkungsmächtiges Muster der abendländischen Literatur vom griechischen Epos über den höfischen Roman bis zum Bildungsroman eingeschrieben. ${ }^{\text {“32 }}$

Der Prozess der Aufnahme des Fremden, die den entfremdeten Mansur zur Selbst(er)findung führen sollte, lässt sich im Roman folgendermaßen verfolgen:

29 Vgl. Hofmann, Michael 2006: a. a. O. S. 13

$30 \quad$ Ebd. S. 13.

$31 \quad$ Konuk, a. a. O., S. 129.

32 Hofmann, a.a.O., S. 17. 
- Die Flucht aus der Heimat, dem Irak;

- die naive Begeisterung für alles Deutsche;

- die anfängliche Zugehörigkeit zu einem Asylantenkreis;

- die Konstruktion einer okzidentalen bzw. deutschen Genealogie;

- die Entflechtung jeglicher orientalischer Elemente und die Abgrenzung von Bekannten;

- die Bemühung, die deutsche Sprache zu lernen;

- $\quad$ die Selbstbenennung als Mansuri von Einsiedler (M 258);

- der Vermählungsversuch mit Lisa;

- die zweite Flucht ins Unbekannte.

Die Selbsterfindung in der Fremde ist $\mathrm{m}$. E. hierdurch als ein öffener Prozess vorzustellen. Mansur kam sich wie ein Phönix vor, der gerade aus der Asche wiederauferstanden war. Ihm wuchsen die ersten Federn, jedoch war es noch zu schwach, um sich in die Lüfte zu erheben. (M 234)

„Mansur, vor allen anderen er, der einst in der stillen Landschaft des Marschlandes gespielt hatte, machte sich zum Empfang des Frühlings bereit. Er zelebrierte seine Verwandlung, ein sanftes Verwachsen der Lebensadern, in dem alles Orientalische verschwand und an seiner Stelle eine frische, zarte Seele, ein junger Sprössling, zum Vorschein kam. In glücklicher Fügung gelang es ihm, sich an neuem, fruchtbarem Bodem anzupflanzen.“ (M 247)

Trotz der Verwurzelungsversuche in der neuen Heimat, blieb die Identitätsfrage brennender als je zuvor:

„Zuletzt stehst du allein, weißt nicht, ob du Babylonier, wie du einmal behauptet hast, oder Sumerer bist, ein Nachfahre der glorreichen Abbasidendynastie oder ein verkommener Assassine. Oder letzlich ein Halbdeutscher, verhaftet in jener versunkenen Zeit, als deinesgleichen zu Tausenden willenlos in den brodelnden Kessel geworfen wurde. Als verspäteter Wanderer kommst du an und verlangst nach Liebe! Wer hat denn hier wen auf dem Gewissen?“ (M 267)

Gegen Ende des Romans erfahren wir, dass die Erinnerung an die Mutter für Mansur wie eine blutende Wunde geworden war. Sie verfolgte ihm in seinen Träumen bzw. Alpträumen und Visionen und peinigte ihn, so dass er sich selbst als Mörder der eigenen Mutter beschuldigte, besonders deshalb, weil alle Rückzugswege bereits abgeschnitten waren. Er geriet in Verzweiflung: 
Selbst(er)findung in der Fremde

„Wachsen deine Flügel oder wirst du ewig ein kriechender Phönix bleiben? Wartest du auf ein Märchen, auf Legenden im Zeitalter der Börsenticker?“ (M 267)

Die Anpassungsversuche Mansurs scheiterten. Er verließ das Ehebett und ergriff noch einmal die Flucht in den mit Schnee bedeckten Wald.

„Wer ist denn dafür verantwortlich, dass du deine Jahre in einen durchlöcherten Topf gesteckt hast? Das alte Ich, das bodenständige, heimatverbundene? Oder dein zweites Selbst, das nichts anderes im Sinn hat als den ursprünglichen Mansur in Stücke zu schlagen und dich zum Fremden macht in einem kalten Land, zu einem Einhorn, das sein Horn an einem Felsen abgestoßen hat? Der Eintopf ist längst gegessen.“ ( M 268)

Mit diesem offenen Schluss endet der Roman. Der anfangs als der Fremde beschriebene Mansur wird auch am Ende des Romans zum Fremden in der freiwillig gewählten Heimat. „Das rettende Gen ${ }^{6{ }^{33} 3}$ konnte ihm nicht retten. „Aber offenbar genügen die Gene alleine doch nicht, um heimatliche Gefühle entwickeln zu können und Wurzeln zu schlagen“"34, stellt Mona Naggar fest. Sie meint, ihm fehle die eigentliche Grundlage für eine neue Identität: „Das Wichtigste ist natürlich bei ihm nicht vorhanden, die Bildung. Dazu gehört, dass man die Geschichte des Landes kennt, die Philosophie, die Musik. ${ }^{\text {"35 }}$

Ich glaube, dass die Bewältigung der Vergangenheit zudem noch notwendig für Mansur ist. Die Schrecken der Vergangenheit halten an und durchkreuzen seine Anpassungsversuche. Mansur hat die Aufgabe, zuerst die Traumata der Diktatur und des Krieges zu verarbeiten. Gleichzeitig hat er auch, anstatt die orientalischen Elemente für Flecken zu halten und von sich wegwaschen zu versuchen, die Aufgabe sie anzuerkennen. In diesem Fall gilt die Maxime: die Negation von etwas bedeutet seine Anwesenheit. Im Hinblick auf Identitäts(er)findung spielt die Anerkennung des Eigenen eine essentielle Rolle.

\footnotetext{
33 Naggar, Mona 2002: Das rettende Gen. Hussain Al-Mozany erzählt von einem irakischen Aussiedler. In: Neue Zürcher Zeitung, 20.6.2002.

34 Ebd.

35 Ebd.
} 
Der freiwillige Verzicht auf das Eigene führt nicht unbedingt $\mathrm{zu}$ einer erfolgreichen Selbsterfindung in der Fremde. Im Gegenteil. Denn das Ende impliziert die Möglichkeit, ein Schicksal in der Diaspora erleiden zu müssen. Dennoch lässt das offene Ende Hoffnung für Mansur übrig. Die Welt würde zu seinem Zufluchtsort. Er müsste weitere Prozesse der Selbstfindung und der Selbsterfindung durchlaufen. Mansur hat $\mathrm{m}$. E. einen Zwischenraum zwischen den Kulturen zu finden und seine Hybridität und die Hybridität des Anderen tatsächlich anzuerkennen. Eine Versöhnung mit der alten Identität und der Erinnerung ist herbeizuführen, damit positive Entwicklungen ermöglicht werden. Hieraus ergibt sich, dass Identität nicht auf ein stabiles Sein festzulegen, sondern als ein ständiges Werden zu betrachten ist.

Fazit

Identität, Selbst(er)findung und Begegnung mit der Fremde stellen ein komplexes Feld dar, durch das sich das Werk Hussain Al-Mozanys bewegt. In diesem Roman versucht Al-Mozany, um es mit Gino Chiellino auszudrücken, ein nicht deutsches Gedächtnis in die deutsche Sprache einzubringen. ${ }^{36}$ Mansur, sein entfremdeter Titelheld, hat eine hybride Identität, die sich aus orientalischen sowie okzidentalen Elementen konstruieren läßt. Die obigen Darlegungen der transkulturellen Aspekte im Roman lassen zum Ergebnis kommen, dass nicht nur Kulturen transkulturell sind, sondern jedes einzelne Individuum. Transkulturalität ist mit Identitätsfindung eng verbunden. Das Erkennen der fremden Elemente in uns ebnet den Weg zum Erkennen von Ähnlichkeiten mit äußeren Fremdheiten. Die Verleugnung des Eigenen billigt eine Selbsterfindung nicht zu. Der bewusste Entschluss, die Ursprungsheimat und deren Sprache zu verlassen, führt im Falle Mansurs zum Verlust der Identität. Die Anpassung an die bis dahin scheinbar Seinde, deutsche Kultur blieb ihm vorenthalten. Der Prozess einer Selbstfindung durch die Selbsterfindung in der Fremde ist noch nicht vollendet und muss weiter fortgesetzt werden. Identitäten sind dementsprechend als Subjektpositionierungen

36 Chiellino, Gino 2002: Ich in Dresden. Eine Poetik. Thelem. Dresden. S. 33f. 
Selbst(er)findung in der Fremde

und Konstrukte zu verstehen. Wichtig dabei ist die Auffassung, dass es sich hier nicht um einen Ist-Zustand, sondern um eine Dynamik handelt.

\section{Primärliteratur}

AL-MoZANY, Hussain 2002: Mansur oder der Duft des Abendlandes. Reclam, Leipzig.

\section{Sekundärliteratur}

AL-MoZANY, Hussain 2011: Parallelwelten. Thelem. Dresden.

BLIOUMI, Aglaia 2002: Interkulturalität und Literatur. Interkulturelle Elemente in Sten Nadolnys Roman „Selim oder Die Gabe der Rede“. In: Blioumi, Aglaia (Hrsg.): Migration und Interkulturalität in neueren literarischen Texten. Iudicium Verlag. München. S. 28-40.

CHIELLINO, Gino 2002: Ich in Dresden. Eine Poetik. Thelem. Dresden.

FLECHSIG, Karl-Heinz 2002: Kulturelle Identität als Lernproblem. In: Globalisierung als Herausforderung der Erziehung.Theorien, Grundlagen, Fallstudien. Christoph Wulf/ Christine Merkel (Hrsg.), Waxmann Verlag, Münster, S. 64-73.

FURGER, Fridolin 2002: Flucht aus Krieg und Diktatur. Hussain Al-Mozany schreibt mit „Mansur oder der Duft des Abendlandes" eine wunderbare Satire auf die deutsche Asyl- und Einbürgerungspraxis. In: Der Bund, 25.5.2002.

HALL, Stuart 1994: Rassismus und kulturelle Identität. Nachdruck in: Konuk, Kader 2001: Identitäten im Prozeß. Literatur von Autorinnen aus und in der Türkei in deutscher, englischer und türkischer Sprache. Literaturwissenschaft in der Blauen Eule. Band 28. Essen.

HALTER, Martin 2002: Spätaussiedler aus Bagdad. Abendland gesucht: Ein Schelmenroman von Hussain Al-Mozany. In: Frankfurter Allgemeine Zeitung, 12.9.2002.

HEYMER, Björn 1994: Biographisch-bibliographisches Kirchenlexikon. Band VII. Nordhausen.

HOFMANN, Michael 2006: Interkulturelle Literaturwissenschaft. Eine Einführung. Wilhelm Fink Verlag. Paderborn.

KONUK, Kader 2001: Identitäten im Prozeß. Literatur von Autorinnen aus und in der Türkei in deutscher, englischer und türkischer Sprache. Literaturwissenschaft in der Blauen Eule. Band 28. Essen.

LÜSEBRINK, Hans-Jürgen 2008: Interkulturelle Kommunikation. Interaktion, Fremdwahrnehmung, Kulturtransfer. 2., aktualisierte und erweiterte Auflage. Verlag J. B. Metzler. Stuttgart/ Weimar.

LÜSEBRINK, Hans-Jürgen 2011: Interkulturelle Kommunikation im Kontext der Globalisierung. Herausforderungen, Konzepte, methodische Ansätze. In: Interkulturelle Kommunikation. 
Perspektiven einer anwendungsorientierten Germanistik. Eckehard Czucka/Eva Neuland (Hrsg.). Iudicium Verlag. München 2011, 227-246.

MAIR, Meinhard 2015: Erzähltextanalyse. Modelle, Kategorien, Parameter. Ibidem Verlag, Stuttgart.

MARTINEZ, Matias/ SCHEFFEL, Michael 2009: Einführung in die Erzähltheorie. 8.Aufl. C.H.Beck, München.

NAGGAR, Mona 2002: Das rettende Gen. Hussain Al-Mozany erzählt von einem irakischen Aussiedler. In: Neue Zürcher Zeitung, 20.6.2002.

NÜNNING, Ansgar 2001: Metzler Lexikon. Literatur- und Kulturtheorie:Ansätze-PersonenGrundbegriffe. 2.überarb.und erw.Aufl., Stuttgart,Weimar.

ÖZDAMAR, Emine Sevgi 2010: Mutter Zunge, Rotbuch.

PFLITSCH, Andreas 2005: Kreidestriche um die Fruchtsamkeit zu narren? Orientierung über den abendländischen Mythos vom Orient. In: Haridi, Alexander (Hrsg.): West-östlicher Seiltanz. Deutsch-arabischer Kulturaustausch im Schnittpunkt Kairo. DAAD. Trio. Bonn. S. 7-13.

REPGEN, Konrad 2005: Was war das Abendland - Eine Begriffsgeschichte, S. 14. In: Haridi, Alexander (Hrsg.): West-östlicher Seiltanz. Deutsch-arabischer Kulturaustausch im Schnittpunkt Kairo. DAAD. Trio. Bonn. 2005. S.14-17.

RÖSCH, Heidi: Migrationsliteratur im interkulturellen Diskurs. Dresden 1998. Online: http://www.fulbright.de/fileadmin/files/togermany/information/2004-

05/gss/Roesch_Migrationsliteratur.pdf. (Stand: 12.9.2015)

SALEH, Arig 2011: Rezeption arabischer Migrationsliteratur in Deutschland. Eine Untersuchung am Beispiel der in Deutschland lebenden syrischen Autoren. Inauguraldissertation zur Erlangung des Grades eines Doktors der Arabistik am Fachbereich Geschichts- und Kulturwissenschaften der Freien Universität Berlin.

SCHMITZ, Walter 2011: Demolierter Orientalismus. Über Hussain Al-Mozany, einen deutschsprachigen Exilschriftsteller aus dem Irak. In: Parallelwelten. Thelem. Dresden. S. 177-209.

SOMMER, Roy 2001: Fictions of Migration. Ein Beitrag zur Theorie und Gattungstypologie des zeitgenössischen interkulturellen Romans in Großbritannien. Wissenschaftlicher Verlag. Trier.

WEIDNER, Stefan 2002: Zwischen Festmahl und Furz. Hussain Al-Mozany sucht den Deutschen im Araber. In: Die Zeit, 23.5.2002.

WEIDNER, Stefan 2003: Begegnung auf halbem Weg. Laudatio auf Hussain Al-Mozany, ChamissoFörderpreis. In: Jahrbuch der Bayerischen Akademie der Schönen Künste, S. 547-550.

WELSCH, Wolfgang 2011: Was ist eigentlich Transkulturalität? Online: http://www2.unijena.de/welsch/tk-1.pdf. (Stand: 12.9.2015) 